\title{
Three-way assessment of long-chain n-3 PUFA nutrition: by questionnaire and matched blood and skin samples
}

\author{
Sarah C. Wallingford ${ }^{1 *}$, Suzanne M. Pilkington ${ }^{1}$, Karen A. Massey ${ }^{2}$, Naser M. I. Al-Aasswad ${ }^{2}$, \\ Torukiri I. Ibiebele ${ }^{3}$, Maria Celia Hughes ${ }^{3}$, Susan Bennett ${ }^{1}$, Anna Nicolaou ${ }^{2}$, Lesley E. Rhodes ${ }^{1}$ \\ and Adèle C. Green ${ }^{1,3}$ \\ ${ }^{1}$ Dermatological Sciences, Inflammation Sciences Research Group, Photobiology Unit, School of Translational Medicine, \\ Manchester Academic Health Sciences Centre, Salford Royal Foundation Hospital, University of Manchester, \\ Manchester M6 8HD, UK \\ ${ }^{2}$ School of Pharmacy and Centre for Skin Sciences, School of Life Sciences, University of Bradford, Bradford, UK \\ ${ }^{3}$ Cancer and Population Studies Group, Queensland Institute of Medical Research, Brisbane, Australia
}

(Submitted 16 January 2012 - Final revision received 18 April 2012 - Accepted 19 April 2012 - First published online 23 May 2012)

\begin{abstract}
The long-chain $n$-3 PUFA, EPA, is believed to be important for skin health, including roles in the modulation of inflammation and protection from photodamage. FFQ and blood levels are used as non-invasive proxies for assessing skin PUFA levels, but studies examining how well these proxies reflect target organ content are lacking. In seventy-eight healthy women (mean age $42 \cdot 8$, range $21-60$ years) residing in Greater Manchester, we performed a quantitative analysis of long-chain $n-3$ PUFA nutrition estimated from a self-reported FFQ ( $n$ 75) and correlated this with $n$-3 PUFA concentrations in erythrocytes $(n$ 72) and dermis $(n$ 39). Linear associations between the three $n$ - 3 PUFA measurements were assessed by Spearman correlation coefficients and agreement between these measurements was estimated. Average total dietary content of the principal long-chain n-3 PUFA EPA and DHA was 171 (sD 168) and 236 (sD 248) mg/d, respectively. EPA showed significant correlations between FFQ assessments and both erythrocyte $(r 0.57, P<0.0001)$ and dermal $(r 0.33, P=0.05)$ levels, as well as between erythrocytes and dermis $(r 0 \cdot 45, P=0.008)$. FFQ intake of DHA and the sum of $n$-3 PUFA also correlated well with erythrocyte concentrations $(r 0.50, P<0.0001 ; r 0.27, P=0.03)$. Agreement between ranked thirds of dietary intake, blood and dermis approached $50 \%$ for EPA and DHA, though gross misclassification was lower for EPA. Thus, FFQ estimates and circulating levels of the dietary long-chain $n-3$ PUFA, EPA, may be utilised as well-correlated measures of its dermal bioavailability.
\end{abstract}

Key words: $\boldsymbol{n}$-3 PUFA: FFQ: Erythrocytes: Skin: Correlations

A wide range of health benefits have been related to the consumption of long-chain n-3 PUFA including improvements in cardiovascular and several inflammatory disorders ${ }^{(1)}$. Evidence also suggests that these $n$ - 3 PUFA, notably EPA (20:5n-3), can benefit skin health, by tempering inflammation and reducing the acute effects of UV radiation such as sunburn, and potentially protecting against longer-term photoageing and photocarcinogenesis ${ }^{(2-6)}$. In the UK, as of 2004, the recommended daily consumption of long-chain $n$-3 PUFA for a healthy adult was increased from $100-200^{(7)}$ to $450 \mathrm{mg}^{(8)}$ as a result of the emergence of new evidence as to the diversity of potential benefits of $n-3$ PUFA $^{(1,8)}$.

EPA and DHA (22:6n-3) are believed to be the most biologically active $n$ - 3 PUFA, their activities including modulation of lipid messengers and transcriptional activation ${ }^{(9,10)}$. These long-chain $n$-3 PUFA compete with the long-chain n-6 PUFA arachidonic acid (AA, 20:4n-6) for metabolism by cyclooxygenases and lipoxygenases, resulting in the production of less pro-inflammatory eicosanoids ${ }^{(11)}$. The respective shortchain $n$ - 3 and $n-6$ PUFA $\alpha$-linolenic acid (ALA, 18:3n-3) and linoleic acid (LA, 18:2n-6) are regarded as essential fatty acids, as they cannot be synthesised endogenously by humans. Ingested ALA is obtained largely from plant sources and can be metabolised endogenously to the longer-chain $n$-3 PUFA ${ }^{(12)}$; however, the conversion of ALA to its longchain metabolites is inefficient: up to 21 and $9 \%$ for EPA and DHA, respectively ${ }^{(13)}$. Thus, the most effective way of naturally obtaining long-chain $n-3$ PUFA is through the consumption of oily fish and seafood ${ }^{(14)}$. In recent years, food products fortified with $n$-3 PUFA, such as milk, margarine, eggs and bread, have also provided other sources of these bioactive fats ${ }^{(15,16)}$.

Abbreviations: AA, arachidonic acid; ALA, $\alpha$-linolenic acid; BHT, butylated hydroxytoluene; LA, linoleic acid.

*Corresponding author: S. C. Wallingford, fax +44161275 5348, email sarah.wallingford@manchester.ac.uk 
Several methods of estimating $n$-3 PUFA nutrition have been described $^{(17)}$. Erythrocytes and plasma are often used to assess $n$-3 PUFA levels, but few other human tissue targets are as easily sampled. FFQ have become useful tools for quantifying nutrient intakes from diet as they are non-invasive and easily implemented into studies of large populations. Studies examining the relationship between $n-3$ PUFA intake estimated from matched FFQ data and blood samples have mostly reported good correlations between these assessments (correlation coefficients between 0.4 and $0 \cdot 6)^{(17)}$. To date, however, there have not been any comparisons of these assessments with concentrations measured in the skin despite the reported relevance of skin $n$-3 PUFA content to photoprotection and potentially other aspects of skin health, as invasive skin assessment (biopsy) is not generally feasible in population studies.

The principal aim of the present study was to perform a three-way matched assessment of EPA nutrition, in order to assess the value of FFQ and erythrocyte measurements as indicators of the skin 'target organ' content. Using a multidisciplinary approach, we quantified $n-3$ PUFA intake from a self-reported FFQ, and correlated this with concentrations measured in erythrocytes and directly in dermal samples from the same adult volunteers in the UK.

\section{Methods}

\section{Study subjects and design}

This analysis used baseline nutritional data collected from participants in a double-blind, randomised, placebo-controlled nutritional study of $n$ - 3 PUFA supplementation. Women who consumed more than two fish meals per week were excluded a priori in order to reduce undue baseline variation in $n$ - 3 PUFA intake among study participants. Eligible participants were healthy white Caucasian women aged 18-60 years with sun-sensitive skin (sun-reactive skin type II, i.e. skin susceptible to sunburn, with minimal tanning) and confirmed Ni-sensitivity (cutaneous allergy, evident most usually to Ni-plated jewellery). Women were excluded if they were pregnant, unable to eat fish or gelatin, took photoactive medication (e.g. non-steroidal anti-inflammatory drugs), consumed n-3 PUFA supplements or more than two meals per week of oily fish, had sunbathed or used a sunbed in the past 3 months, or reported a history of atopy, skin cancer, photosensitivity disorder or cardiac disease. Participants were recruited through the Contact Dermatitis Investigation Unit of Salford Royal NHS Foundation Hospital, Manchester, UK or by advertisements placed in the hospital, the University of Manchester and local newspapers. This study was conducted according to the guidelines laid down in the Declaration of Helsinki and all procedures involving human subjects were approved by the North Manchester Research Ethics Committee. Written informed consent was obtained from all subjects.

\section{Dietary assessment}

Dietary intake was assessed using a self-administered, semiquantitative FFQ modified from the validated Nurses' Health
Study questionnaire (USA) ${ }^{(18)}$ and the Nambour Skin Cancer Study questionnaire (Australia) ${ }^{(19,20)}$. Only minor modifications were made to the Australian version (validated for PUFA intake using the method of triads ${ }^{(20)}$ ) in order to ensure that the FFQ used in our present study reflected UK dietary components. There were 147 food items on the questionnaire and participants were asked to report the frequency with which a specified serving of each food item was consumed. Serving sizes were expressed either as standard measurements (i.e. one cup, one teaspoon, etc.) or in natural units (i.e. one egg, one slice, etc.). Frequency options ranged from 'never' to ' $4+$ times per $d$ '. Additional questions collected information on trimming of visible fat from meat, types of fats and oils used on foods and in cooking, as well as on specific foods consumed that were fortified with n-3 PUFA. Questions regarding self-administered dietary supplements were also included.

Participants reported the average frequency with which they consumed food items in the past 6 months. Average daily intake was calculated by expressing the frequency response of a food item as a proportion of daily use, multiplied by the serving size and by the nutrient content of the food. The McCance and Widdowson's Composition of Foods Integrated Dataset was used to obtain nutrient compositions for UK food items ${ }^{(21)}$. When nutrients could not be obtained from the McCance 2002 database, items were selected from Australian databases ${ }^{(22-24)} \cdot n-3$ PUFA contents of additional foods including margarine/spreads, bread and eggs were obtained from manufacturers' information.

In addition to the FFQ, women were asked regarding their height and weight for estimation of BMI and about their current or former smoking status.

\section{Biological sampling}

Blood samples were taken from the antecubital fossa of participants and collected in EDTA monovette tubes (Sarsstedt) and centrifuged for $15 \mathrm{~min}$ at $1500 \mathrm{rpm}$; the erythrocyte fractions were collected and stored at $-70^{\circ} \mathrm{C}$ until analysis. A random half of the study subjects had $5 \mathrm{~mm}$ punch skin biopsies taken from an unexposed site of upper buttock skin under local anaesthesia (2\% lignocaine without adrenaline). Skin biopsies were snap-frozen in liquid $\mathrm{N}_{2}$ and stored at $-70^{\circ} \mathrm{C}$ for analysis.

\section{Erythrocyte and skin biopsy analysis}

Skin samples were defrosted on ice. Only dermal tissue samples were available for this analysis and these were incubated in chloroform-methanol $(4 \mathrm{ml})(2: 1, \mathrm{v} / \mathrm{v})$ containing butylated hydroxytoluene (BHT) $(0.01 \%(\mathrm{w} / \mathrm{v})$ overnight at $4^{\circ} \mathrm{C}$ and homogenised using a blade homogeniser. Lipids were extracted using a further two volumes of chloroformmethanol-BHT as described before ${ }^{(25)}$. Erythrocyte samples $(1 \mathrm{ml})$ were defrosted on ice and lipids were also extracted with chloroform-methanol-BHT $(4 \mathrm{ml})$. The solvent was removed under $\mathrm{N}_{2}$ and the lipid extract was stored at $-20^{\circ} \mathrm{C}$ before analysis. 
Fatty acid methyl esters were prepared using $\mathrm{BF}_{3}$ in methanol, and analysed by GC (GC-flame ionisation detector) on a BPX70 GC capillary column (SGE Europe Limited), using $\mathrm{He}$ as the carrier gas. Heneicosaenoic acid (C21:0) was used as the internal standard (ACS reagent, Sigma-Aldrich) with a thirty-seven fatty acid methyl esters mixed standard (Supelco), the reference for identification of fatty acids. The detection limit for this method was $5 \mu \mathrm{g}$ per sample, and all measured $n$-3 PUFA levels were well above this lower boundary. Results for both erythrocytes and dermis were expressed as percentage of total fatty acids in each sample. The PUFA examined included the principal long-chain n-3 PUFA EPA and DHA, the principal short-chain $n-3$ PUFA ALA, and the principal long- and short-chain n-6 PUFA, AA and LA, respectively.

\section{Statistical analyses}

Energy intakes from the FFQ were assessed to identify any women with extreme or implausible values $(>14700$ and $<2100 \mathrm{~kJ} / \mathrm{d}$ ) using Willett's criteria ${ }^{(26)}$. Fatty acid intakes were also adjusted for energy intake using the residual method $^{(18)}$. Spearman correlation coefficients for each PUFA were used to assess the linear relationship between each of the parameters (FFQ and erythrocytes, FFQ and dermis, erythrocytes and dermis). Significance was set at $P<0 \cdot 05$. Correlations were also performed stratified by age (21-40; 41-60 years $)$, and, when available, by BMI $\left(<25, \geq 25 \mathrm{~kg} / \mathrm{m}^{2}\right)$ and by smoking status (never or ever smoked) to assess possible effect modification by these factors. Agreement between the PUFA measurements, in ranked thirds, from FFQ, erythrocytes and dermis was assessed by comparing percentage exact agreement and gross misclassification (disagreement across two tertiles) to random expected values. All statistical analyses were performed using Statistical Analysis Systems software package version 9.2 (SAS Institute).

\section{Results}

A total of seventy-nine eligible volunteers were enrolled for the study, one woman withdrew, and baseline information for analysis was thus obtained from seventy-eight volunteers, with a mean age of 42.8 (SD 10.0) years (range 21-60 years) and an average BMI (available for sixty-one women) of $26.7(\mathrm{sD} 5 \cdot 0) \mathrm{kg} / \mathrm{m}^{2}$ (range $19 \cdot 4-44.2 \mathrm{~kg} / \mathrm{m}^{2}$ ) (Table 1). Of the seventy-eight participants, seventy-five completed and returned their FFQ, and, of these, four people were omitted from further FFQ-related analyses as they had implausibly high energy intakes. The mean daily energy intake of the remaining participants ( $n$ 71) was 7889 (SD 2390) kJ. Overall, seventy-two participants provided blood samples, and all thirty-nine volunteers randomised to providing skin samples did so. Mean values and standard deviations were calculated for total and specific n-3 PUFA intakes using FFQ data (energy adjusted), blood samples and skin biopsies (Table 2). Average total EPA, DHA and ALA intake from FFQ was 171 (SD 168), 236 (SD 243) and 850 (sD 260) $\mathrm{mg} / \mathrm{d}$. Energy-unadjusted means from FFQ were similar (data not shown). From the tissue measures, mean EPA and DHA (percentage of total fatty acids) were highest in erythrocytes, while mean ALA was highest in dermis.

Several significant correlations were observed between the amount of long-chain $n$-3 PUFA in FFQ, erythrocytes and dermis. FFQ data for EPA showed the most substantial significant correlation coefficients with erythrocytes and dermal data ( $r$ 0.57, $P<0.0001$ and $r 0.33, P=0.05$, respectively) (Table 3; Fig. $1(\mathrm{a}-\mathrm{c}))$. We observed a significant correlation between FFQ and erythrocytes for DHA $(r 0.50, P<0.0001)$ and for the sum total of EPA + DHA + ALA $(r 0 \cdot 27, P=0 \cdot 03)$ (Table 3; Fig. $2(\mathrm{a}-\mathrm{c}))$. None of the three-way assessments showed significant correlations for ALA. The only significant inverse correlation observed was between erythrocytes and dermal

Table 1. Baseline population characteristics of women recruited for double-blind, randomised, placebo-controlled nutritional study $(n 78)$

(Mean values, standard deviations, ranges, number of women and percentages)

\begin{tabular}{|c|c|c|c|}
\hline Demographics & Mean & SD & Range \\
\hline Age (years) ${ }^{\star}$ & $42 \cdot 8$ & $10 \cdot 0$ & $21-60$ \\
\hline $\mathrm{BMI}\left(\mathrm{kg} / \mathrm{m}^{2}\right) \dagger$ & $26 \cdot 7$ & $5 \cdot 0$ & $19 \cdot 4-44 \cdot 2$ \\
\hline \multicolumn{4}{|l|}{ Smoking status $\dagger$} \\
\hline \multicolumn{4}{|l|}{ Never smoked } \\
\hline$n$ & & & 30 \\
\hline$\%$ & & & 39 \\
\hline \multicolumn{4}{|l|}{ Ex-smoker } \\
\hline$n$ & & & 18 \\
\hline$\%$ & & & 23 \\
\hline \multicolumn{4}{|l|}{ Current smoker } \\
\hline$n$ & & & 13 \\
\hline$\%$ & & & 17 \\
\hline Pack years (ex/current smokers) $†$ & $6 \cdot 9$ & $11 \cdot 3$ & $0 \cdot 25-56$ \\
\hline \multicolumn{4}{|l|}{ Diet } \\
\hline \multicolumn{4}{|l|}{ Nutrient intakes $(g / d) \ddagger \S$} \\
\hline Protein & $84 \cdot 6$ & $12 \cdot 9$ & $54 \cdot 3-121 \cdot 6$ \\
\hline Fat & $65 \cdot 8$ & $12 \cdot 6$ & $35 \cdot 2-96 \cdot 8$ \\
\hline SFA & $27 \cdot 0$ & $6 \cdot 2$ & $11 \cdot 2-44 \cdot 3$ \\
\hline MUFA & 22.5 & 4.8 & $10 \cdot 3-33.9$ \\
\hline PUFA & 9.5 & 3.3 & $5 \cdot 6-22 \cdot 2$ \\
\hline Carbohydrate & 211.5 & $31 \cdot 7$ & $138 \cdot 7-293 \cdot 2$ \\
\hline Alcohol $(\mathrm{g} / \mathrm{d})$ & $10 \cdot 8$ & $18 \cdot 9$ & $0.00006-129.2$ \\
\hline \multicolumn{4}{|l|}{ Use supplementsł } \\
\hline$n$ & \multicolumn{3}{|r|}{11} \\
\hline$\%$ & \multicolumn{3}{|r|}{15} \\
\hline \multicolumn{4}{|l|}{ Do not eat meatł } \\
\hline$n$ & \multicolumn{3}{|r|}{6} \\
\hline$\%$ & \multicolumn{3}{|r|}{8} \\
\hline \multicolumn{4}{|l|}{ Use $n$-3 PUFA fortified $\ddagger$} \\
\hline \multicolumn{4}{|l|}{ Margarine } \\
\hline$n$ & \multicolumn{3}{|r|}{17} \\
\hline$\%$ & \multicolumn{3}{|r|}{23} \\
\hline \multicolumn{4}{|l|}{ Bread } \\
\hline$n$ & \multicolumn{3}{|r|}{5} \\
\hline$\%$ & \multicolumn{3}{|r|}{7} \\
\hline \multicolumn{4}{|l|}{ Eggs } \\
\hline$n$ & \multicolumn{3}{|r|}{9} \\
\hline$\%$ & \multicolumn{3}{|r|}{12} \\
\hline \multicolumn{4}{|l|}{ Milk } \\
\hline$n$ & \multicolumn{3}{|r|}{2} \\
\hline$\%$ & \multicolumn{3}{|r|}{3} \\
\hline
\end{tabular}

${ }^{*}$ Missing data $n 5(6 \%)$.

† Missing data $n 17(22 \%)$.

$\ddagger$ Missing data $n 3(4 \%)$.

$\S$ Mean daily nutrient intake values energy-adjusted and exclude people with values $>14700 \mathrm{~kJ}(n)$. 
Table 2. Principal long- and short-chain n-3 PUFA assessed from FFQ, erythrocytes and dermal tissue

(Mean values and standard deviations)

\begin{tabular}{|c|c|c|c|c|c|c|}
\hline \multirow[b]{2}{*}{ Nutrient } & \multicolumn{2}{|c|}{$\mathrm{FFQ}^{*}(n 71)$} & \multicolumn{2}{|l|}{ Erythrocytes ( $n 72)$} & \multicolumn{2}{|l|}{ Dermis ( $n$ 39) } \\
\hline & Mean $(\mathrm{mg} / \mathrm{d})$ & SD & Mean (percentage of total FA) & SD & Mean (percentage of total FA) & SD \\
\hline EPA $(20: 5 n-3)$ & 171 & 168 & 0.93 & 0.43 & 0.07 & 0.03 \\
\hline DHA $(22: 6 n-3)$ & 236 & 243 & 3.56 & 0.95 & 0.18 & 0.09 \\
\hline $\operatorname{ALA}(18: 3 n-3)$ & 850 & 260 & 0.40 & 0.31 & 0.74 & 0.24 \\
\hline $\mathrm{EPA}+\mathrm{DHA}+\mathrm{ALA} \dagger$ & 1256 & 501 & 4.90 & 1.31 & 0.99 & 0.29 \\
\hline
\end{tabular}

FA, fatty acid; ALA, $\alpha$-linolenic acid.

*Intakes from FFQ energy-adjusted.

† Sum of EPA, DHA and ALA.

$\mathrm{EPA}+\mathrm{DHA}+\mathrm{ALA}(r-0 \cdot 39, P=0 \cdot 02)$. When the influence of age, body weight and smoking status on FFQ and erythrocyte correlations (there was an insufficient number of dermal samples to permit stratified analyses) was assessed, correlations remained relatively consistent across categories for EPA and DHA. FFQ and erythrocyte correlations for the sum of the $n-3$ PUFA fluctuated according to age $(<40$ years or $>40$ years $)$, BMI $\left(<25\right.$ or $\left.\geq 25 \mathrm{~kg} / \mathrm{m}^{2}\right)$ and smoking habit (ever or never), but only changed by age group for ALA (not shown).

When agreement between ranked thirds of $n$ - 3 PUFA nutrition by FFQ, erythrocytes and dermis was assessed (Table 4), EPA had the best agreement between all measures, with over half of subjects classified into the exact third of EPA intake and low gross misclassification. Agreement between ranked thirds of DHA also approached $50 \%$, though a higher proportion of women were misclassified. Overall, assignment to the same or adjacent ranked third category of EPA and DHA was 79-94\%. Exact agreement was lower than expected for the sum of the $n$-3 PUFA and ALA.

All analyses were repeated for LA, AA, the sum of LA + AA and the ratio of total $n-6: n-3$. There were no significant correlations between the $n-6$ PUFA measures, and only the FFQ and erythrocyte correlation was significant for $n-6: n-3$ ( $r$ 0.42, $P=0.0004)$. Exact agreement of ranked thirds and gross misclassification were highly variable (not shown).

\section{Discussion}

In the present study, we have demonstrated that the assessment of dietary EPA intake using an FFQ, and corresponding circulating levels, are in turn significantly and well correlated with the measurement of dermal content. These findings are consistent with evidence of the functional significance of EPA in $\operatorname{skin}^{(4,6,27)}$. This is important new knowledge for those concerned with promotion of skin health through improved nutrition, since previous studies have not examined these parameters synchronously, and confirms the importance of dietary intake of EPA and DHA on their bioavailability in a clinically relevant target tissue.

Among female study populations similar to ours, average daily intakes of EPA and DHA have been reported as 61-280 and $109-460 \mathrm{mg} / \mathrm{d}$, respectively, from $\mathrm{FFQ}^{(28-31)}$; the FFQ intakes measured in our present study fell within these ranges. Our mean ALA intakes were only slightly lower than those reported previously $(1030-1200 \mathrm{mg} / \mathrm{d})^{(28,30)}$. Current recommended daily intake of total long-chain $n-3$ PUFA for healthy adults is $450 \mathrm{mg}$ in the $\mathrm{UK}^{(8)}$. The relatively low intake of EPA and DHA by the women in this study (half the UK recommendation) might be partly explained by the eligibility criterion of consuming less than two serves of fatty fish a week. In addition, consumption of fatty fish in general has declined markedly in the past century and, although a comparatively minor source of $n-3$ PUFA, lamb and beef consumption in the UK has also declined over the past 20 years ${ }^{(32)}$; thus these trends may also have contributed to the women's lower-than-recommended $n$-3 PUFA consumption.

Various methods have been used for the assessment of bioavailable $n$-3 PUFA including sampling of erythrocytes, platelets, plasma, serum, cholesterol esters, phospholipids ${ }^{(28,33,34)}$, adipose tissue ${ }^{(31,35,36)}$ and buccal mucosa ${ }^{(37)}$. Erythrocyte measurements are considered better markers of circulating

Table 3. Spearman correlation coefficients for principal long- and short-chain $n-3$ PUFA assessed from FFQ, erythrocytes and dermal tissue

\begin{tabular}{|c|c|c|c|c|c|c|}
\hline \multirow[b]{2}{*}{ Nutrient } & \multicolumn{2}{|c|}{$\begin{array}{l}\text { FFQ } v \text {. erythro- } \\
\text { cytes }(n 65)\end{array}$} & \multicolumn{2}{|c|}{$\begin{array}{c}\text { FFQ } v \text {. dermis } \\
(n \text { 35) }\end{array}$} & \multicolumn{2}{|c|}{$\begin{array}{c}\text { Erythrocytes } v . \\
\text { dermis }(n 34)\end{array}$} \\
\hline & $r$ & $P$ & $r$ & $P$ & $r$ & $P$ \\
\hline EPA $(20: 5 n-3)$ & 0.57 & $<0.0001^{*}$ & 0.33 & $0.05^{\star}$ & 0.45 & $0.008^{*}$ \\
\hline DHA (22:6n-3) & 0.50 & $<0.0001^{*}$ & 0.18 & 0.29 & 0.09 & 0.62 \\
\hline ALA $(18: 3 n-3)$ & 0.04 & 0.78 & -0.13 & 0.44 & -0.01 & 0.94 \\
\hline $\mathrm{EPA}+\mathrm{DHA}+\mathrm{ALA} \dagger$ & 0.27 & $0.03^{\star}$ & -0.18 & 0.30 & -0.39 & $0.02^{*}$ \\
\hline
\end{tabular}

ALA, $\alpha$-linolenic acid.

* Significant correlation $(P<0.05)$

† Sum of EPA, DHA and ALA. 

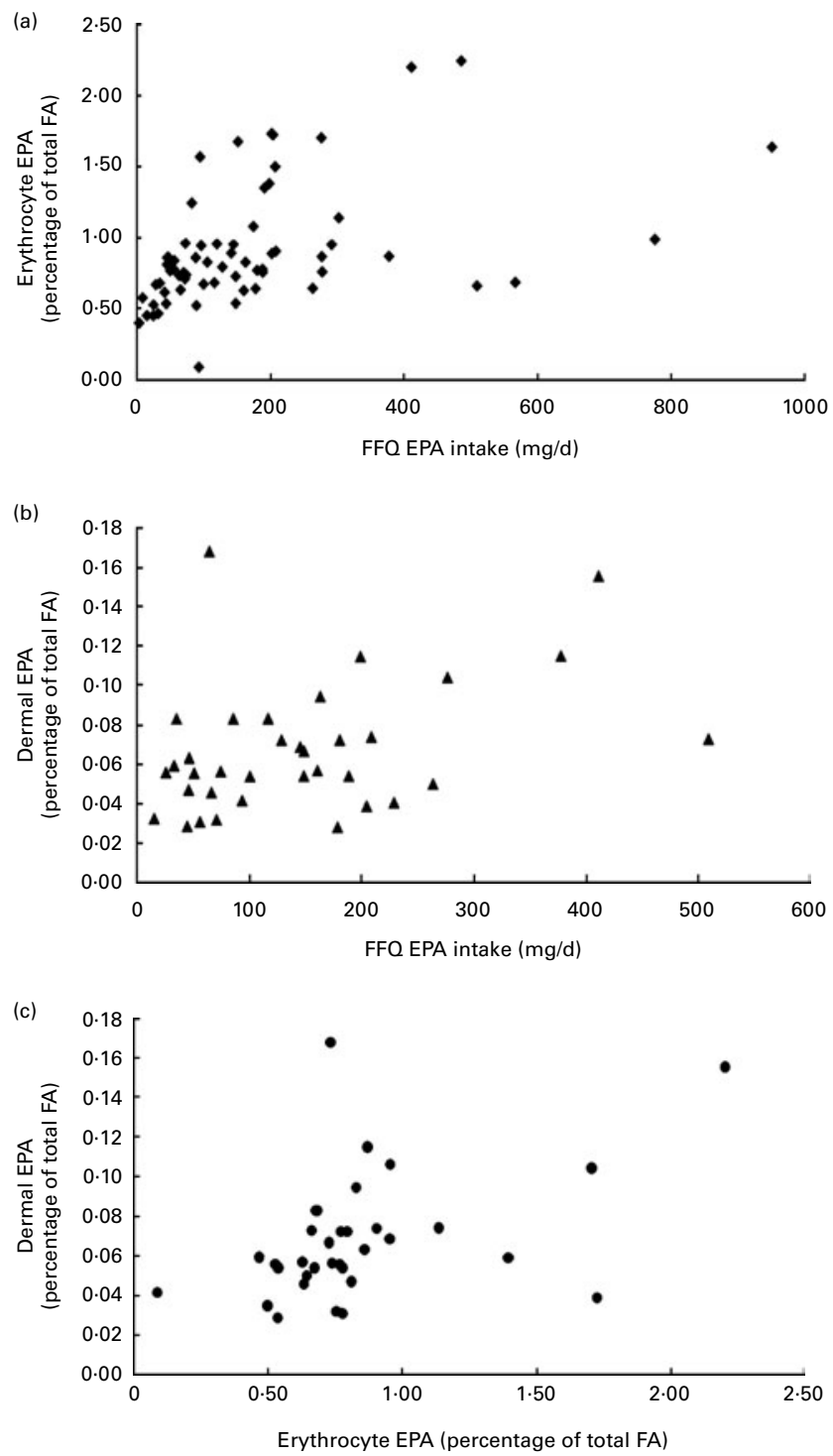

Fig. 1. Bivariate plots of EPA levels from (a) FFQ (mg/d) $v$. erythrocytes (percentage of total fatty acid (FA)), (b) FFQ $v$. dermis (percentage of total FA) and (c) erythrocytes $v$. dermis, with Spearman correlation coefficients and $P$ values. (a) $r 0.57, P<0.0001$; (b) $r 0.33, P<0.05$ and (c) $r 0.45, P<0.008$.

blood PUFA levels than serum or plasma measurements, as they reflect an intake period of several weeks and contain a high proportion of $\mathrm{PUFA}^{(33)}$. Of the studies reporting on the validity of FFQ in reference to blood concentrations, only a few reported erythrocyte data as opposed to serum or plasma phospholipid fatty acids. Our correlation coefficients for intake from FFQ compared with erythrocyte concentrations were slightly higher than those previously reported (0.37-0.40 and 0.16-0.39) for EPA and DHA, respectively $^{(29,38)}$, and consistent with the high validity coefficients for FFQ and erythrocyte measures of long-chain n-3 PUFA observed in the analysis of Swierk et al. ${ }^{(39)}$. The apparent tighter fit of the correlations at lower levels and dispersion at higher levels in the correlation figures was probably due to the study exclusion criteria recruiting a population of relatively low $n$-3 PUFA consumers. Few participants consumed high levels of $n-3$ PUFA such that these data points appear more as relative outliers. That a correlation was not observed in the present study between FFQ and blood ALA content is probably due to the estimated $30 \%$ lost in metabolic oxidation of dietary ALA into carbon dioxide for energy ${ }^{(13)}$.

Detailed descriptions of $n$-3 PUFA content of human skin are sparse in the literature, and the currently reported series appears by far the largest to date. Previous smaller human volunteer studies have reported a low skin content of EPA, ranging from $0.05 \% \mathrm{~mol}$ in whole-skin biopsies $(n-14)^{(27)}$ to $1 \cdot 1 \% \mathrm{~mol}$ in skin-shave biopsies comprising largely epidermis $(n 6)^{(2)}$. The present findings are consistent with these reports, the dermis exhibiting an EPA content within this range. The lead-time for dietary $n-3$ PUFA to affect skin content is
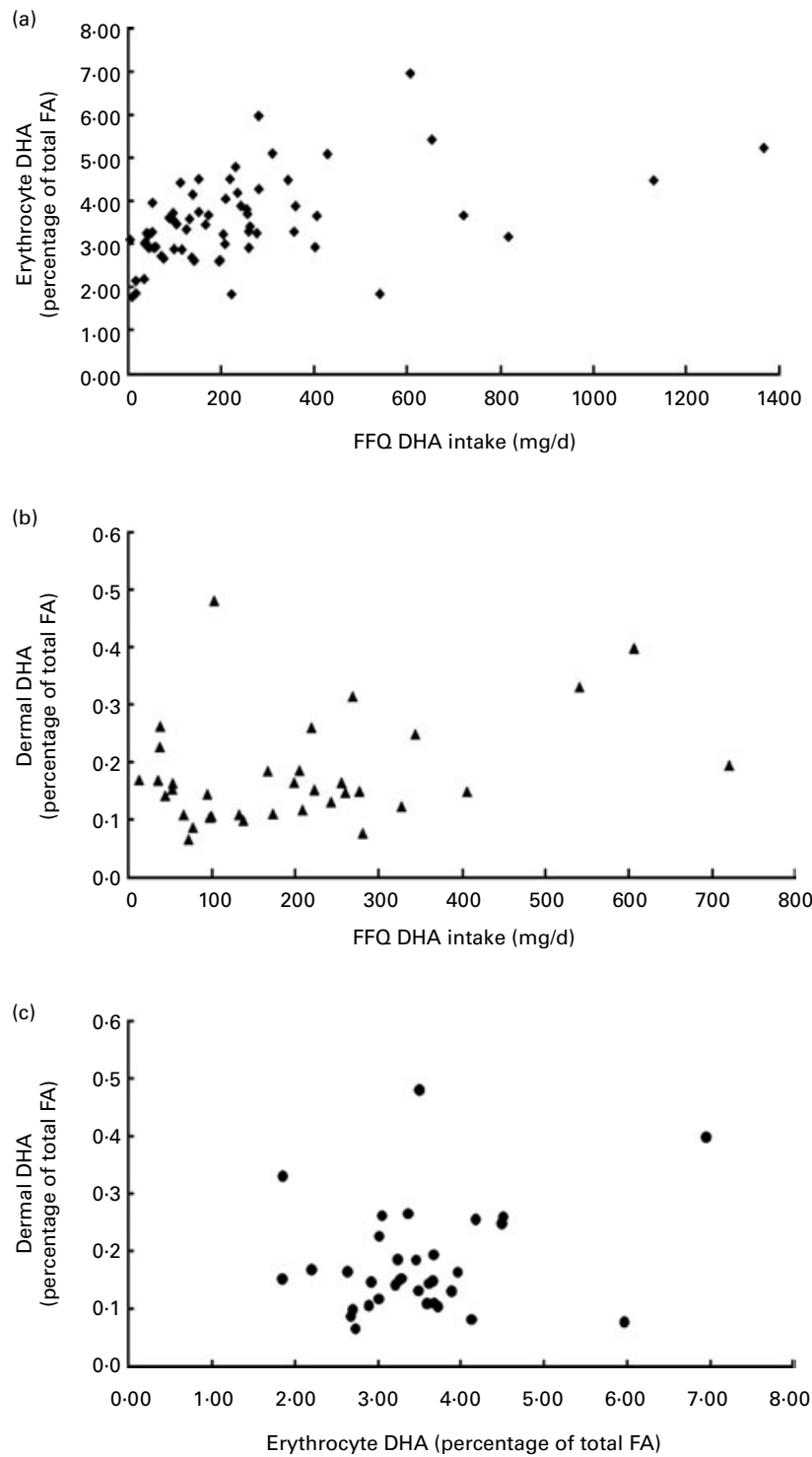

Fig. 2. Bivariate plots of DHA levels from (a) FFQ (mg/d) $v$. erythrocytes (percentage of total fatty acid (FA)), (b) FFQ $v$. dermis (percentage of total FA) and (c) erythrocytes $v$. dermis, with Spearman correlation coefficients and $P$ values. (a) $r 0.50, P<0.0001$; (b) $r 0.18, P<0.29$ and (c) $r 0.09, P<0.62$. 
Table 4. Percentage agreement between tertile assignments according to principal long- and short-chain $n-3$ PUFA assessed from FFQ, erythrocytes and dermal tissue

\begin{tabular}{|c|c|c|c|c|c|c|c|c|c|}
\hline \multirow[b]{2}{*}{ Nutrient } & \multicolumn{3}{|c|}{ FFQ $v$. erythrocytes ( $n$ 65) } & \multicolumn{3}{|c|}{ FFQ $v$. dermis $(n 35)$} & \multicolumn{3}{|c|}{ Erythrocytes $v$. dermis $(n 34)$} \\
\hline & Exact $(\%)$ & \pm 1 Tertile $(\%)$ & GM (\%) & Exact (\%) & \pm 1 Tertile $(\%)$ & GM (\%) & Exact (\%) & \pm 1 Tertile $(\%)$ & GM (\%) \\
\hline EPA $(20: 5 n-3)$ & 48 & 46 & 6 & 54 & 29 & 17 & 53 & 41 & 6 \\
\hline DHA $(22: 6 n-3)$ & 43 & 49 & 8 & 29 & 57 & 14 & 50 & 29 & 21 \\
\hline ALA $(18: 3 n-3)$ & 29 & 49 & 22 & 37 & 34 & 29 & 32 & 47 & 21 \\
\hline $\mathrm{EPA}+\mathrm{DHA}+\mathrm{ALA}^{*}$ & 32 & 58 & 9 & 31 & 40 & 29 & 24 & 41 & 35 \\
\hline Random expected & 33 & 45 & 22 & 33 & 45 & 22 & 33 & 45 & 22 \\
\hline
\end{tabular}

GM, gross misclassification; ALA, $\alpha$-linolenic acid.

* Sum of EPA, DHA and ALA.

uncertain, but it has been observed that daily supplementation with $4 \mathrm{~g}$ EPA capsules resulted in an 8-fold increase in skin content of EPA after 3 months ${ }^{(27)}$. Our findings complement these results by demonstrating that even low dietary EPA intake appears to be reflected in the skin.

Human $n$-3 PUFA supplementation studies have additionally demonstrated the role of EPA in decreasing the skin's UV-induced inflammatory response. Specifically, EPA has been shown to increase the skin's threshold to sunburn erythema, and to reduce both basal and UV-induced cutaneous levels of the eicosanoid $\mathrm{PGE}_{2}{ }^{(2-6,27,40)}$, a key mediator of the erythema and also involved in skin cancer promotion $^{(41)}$. This is consistent with the known competition of EPA with AA for metabolism by cyclo-oxygenase, leading to reduced $\mathrm{PGE}_{2}$ production ${ }^{(9)}$. A range of human studies also supports that $n$ - 3 PUFA may protect against the longerterm photodamage of skin carcinogenesis and ageing ${ }^{(4,27,42)}$. The recent revision of dietary guidelines to intake of $450 \mathrm{mg}$ long-chain $n$-3 PUFA per d was done to align with existing recommendations of two weekly servings of fatty fish $^{(8)}$; however, the adequacy of these new dietary recommendations for skin health remains unknown. Little is known about the threshold $n$-3 PUFA intake required for protection against adverse skin health outcomes. This requires examination in future studies, particularly in view of the continued escalation in skin cancer incidence in many white-skinned populations ${ }^{(43)}$; a dietary approach to prevention could have widespread impact at a population level ${ }^{(5)}$.

The present study's limitations include potential for error in the accuracy of PUFA intake measurements from self-reported FFQ due to issues of recall by the participants. Further, although a similar version of the FFQ was validated in an Australian population ${ }^{(20)}$, the updated FFQ may not be fully valid when used in our UK-based population. We also acknowledge that, as a dietary measurement tool, any FFQ may not be fully valid and reliable ${ }^{(44)}$. On the other hand, the McCance database ${ }^{(21)}$, used to identify the nutrient composition of food items in this study, is the database most widely accepted and used in the UK and as such provides the most accurate PUFA data available for the intake frequencies provided from the FFQ. A limitation of our dermal PUFA data was that a large amount of variation remained unaccounted for when comparing these data with FFQ intake and erythrocyte content. Metabolic differences between individuals in both uptake and transport of $n-3$ PUFA to the skin may explain some of this variation, but future investigative studies are needed in order to address this issue. Furthermore, the variability of $n-3$ PUFA levels in skin from different anatomic body sites remains unknown, although our EPA data were consistent with previous reports ${ }^{(2,27)}$. The number of skin samples was also insufficient to precisely evaluate how age, BMI and smoking habits may influence dermal $n$-3 PUFA content in relation to dietary content. Finally, our present study participants were all females; so it is not known how our results apply to males, and since study participants were volunteers, their representativeness of similarly aged healthy UK women is unknown.

In summary, concentrations of EPA in dermal and erythrocyte samples from these healthy women showed significant correlations with EPA consumption, demonstrating that FFQ intake estimates provided a good measure of both the circulating and skin bioavailability of this long-chain n-3 PUFA. Further research in a more diverse population is required to extend these findings and to determine the threshold of $n-3$ PUFA intake required to sustain skin benefit.

\section{Acknowledgements}

The authors are grateful to the volunteers who participated in the present study. This study was funded by a grant from the Association for International Cancer Research (L. E. R. and A. N., no. 08-0131). S. C. W. and A. C. G. were supported by a fellowship from the Medical Research Council, UK (no. 89912). None of the authors had a personal or financial conflict of interest. The authors' contributions to the study were as follows: S. C. W. managed the data, performed statistical analysis and wrote the manuscript; S. M. P. assisted with data collection and wrote the manuscript; K. A. M. and N. M. I. A.-A. carried out laboratory analyses; T. I. I. and M. C. H. assisted with data management and statistical analysis and wrote the manuscript; S. B. recruited volunteers and collected the data; A. N. supported laboratory analyses and manuscript writing; L. E. R. designed the study and wrote the manuscript; A. C. G. assisted with interpretation of the results and writing of the manuscript.

\section{References}

1. Ruxton CHS, Reed SC, Simpson MJA, et al. (2004) The health benefits of omega- 3 polyunsaturated fatty acids: a review of the evidence. J Hum Nutr Diet 107, 449-459. 
2. Rhodes LE, O'Farrell S, Jackson MJ, et al. (1994) Dietary fishoil supplementation in humans reduces UVB-erythemal sensitivity but increases epidermal lipid peroxidation. J Invest Dermatol 103, 151-154.

3. Hakim IA, Harris RB \& Ritenbaugh C (2000) Fat intake and risk of squamous cell carcinoma of the skin. Nutr Cancer 36, 155-162.

4. Kim HH, Cho S, Lee S, et al. (2006) Photoprotective and antiskin-aging effects of eicosapentaenoic acid in human skin in vivo. J Lipid Res 47, 921-930.

5. Black HS \& Rhodes LE (2006) The potential of omega-3 fatty acids in the prevention of non-melanoma skin cancer. Cancer Detect Prev 30, 224-232.

6. Pilkington SM, Watson REB, Nicolaou A, et al. (2011) Omega-3 polyunsaturated fatty acids: photoprotective macronutrients. Exp Dermatol 20, 537-543.

7. Cardiovascular Review Group (1994) Committee on Medical Aspects of Food Policy. Nutritional Aspects of Cardiovascular Disease. Report on Health and Social Subjects. London: Department of Health.

8. Scientific Advisory Committee on Nutrition (2004) Advice for Fish Consumption: Benefits, Risks. London: Food Standards Agency.

9. Ziboh VA, Miller CC \& Cho Y (2000) Metabolism of polyunsaturated fatty acids by skin epidermal enzymes: generation of antiinflammatory and antiproliferative metabolites. $\mathrm{Am} \mathrm{J}$ Clin Nutr 71, 361S-366S.

10. Deckelbaum RJ, Worgall TS \& Seo T (2006) $n$-3 Fatty acids and gene expression. Am J Clin Nutr 83, S1520-S1525.

11. Massey K \& Nicolaou A (2011) Lipidomics of polyunsaturated-fatty-acid-derived oxygenated metabolites. Biochem Soc Trans 39, 1240-1246.

12. Pilkington SM \& Rhodes LE (2010) Omega-3 fatty acids and skin. In Nutrition for Healthy Skin: Strategies for Cosmetic and Clinical Practice [J Krutmann and P Humbert, editors]. Heidlberg/New York: Springer.

13. Burdge GC \& Wootton SA (2002) Conversion of $\alpha$-linolenic acid to eicosapentaenoic, docosapentaenoic and docosahexaenoic acids in young women. BrJ Nutr 88, 411-420.

14. Whelan J \& Rust C (2006) Innovative dietary sources of $n-3$ fatty acids. Ann Rev Nutr 26, 75-103.

15. Metcalf RG, James MJ, Mantzioris E, et al. (2003) A practical approach to increasing intakes of $n-3$ polyunsaturated fatty acids: use of novel foods enriched with $n-3$ fats. Eur J Clin Nutr 57, 1605-1612.

16. Shapira N, Weill P \& Loewenbach R (2008) Egg fortification with $n-3$ polyunsaturated fatty acids (PUFA): nutritional benefits versus high $n-6$ PUFA western diets, and consumer acceptance. Isr Med Assoc J 10, 262-265.

17. Øverby NC, Serra-Majem L \& Andersen LF (2009) Dietary assessment methods on $n-3$ fatty acid intake: a systematic review. Br J Nutr 102, S56-S63.

18. Willett WC, Sampson L, Stampfer MJ, et al. (1985) Reproducibility and validity of a semiquantitative food frequency questionnaire. Am J Epidemiol 122, 51-65.

19. Marks GC, Hughes MC \& van der Pols JC (2006) The effect of personal characteristics on the validity of nutrient intake estimates using a food-frequency questionnaire. Public Health Nutr 9, 394-402.

20. McNaughton SA, Hughes MC \& Marks GC (2007) Validation of a FFQ to estimate the intake of PUFA using plasma phospholipid fatty acids and weighed foods records. BrJ Nutr $\mathbf{9 7}$, 561-568.

21. McCance W (2002) The Composition of Foods Integrated Dataset, 6th ed. London: Food Standards Agency.
22. Mann N, Sinclair AJ, Percival P, et al. (2003) Development of a database of fatty acids in Australian food. Nutr Dietetics 60 , $42-45$.

23. Australia New Zealand Food Authority (1999) Supplement to NUTTAB 95: Nutrient Content Database. Canberra: ANZFA.

24. Food Standard Australia New Zealand (2007) NUTTAB 2006 - Australian Food Composition Tables. Canberra: FSANZ.

25. Green P, Anyakoha N, Yadid G, et al. (2009) Arachidonic acid-containing phosphatidylcholine species are increased in selected brain regions of a depressive animal model: implications for pathophysiology. Prostagland Leuko Essent Fatty Acids 80, 213-220.

26. Willett WC (1998) Nutritional Epidemiology. New York, NY: Oxford University Press.

27. Rhodes LE, Shahbakhti H, Azurdia RM, et al. (2003) Effect of eicosapentaenoic acid, an omega- 3 polyunsaturated fatty acid, on UVR-related cancer risk in humans. An assessment of early genotoxic markers. Carcinogenesis 24, 919-925.

28. Andersen L, Solvoll K \& Drevon C (1996) Very-long-chain $n-3$ fatty acids as biomarkers for intake of fish and $n-3$ fatty acid concentrates. Am J Clin Nutr 64, 305-311.

29. Sullivan BL, Williams PG \& Meyer BJ (2006) Biomarker validation of a long-chain omega-3 polyunsaturated fatty acid food frequency questionnaire. Lipids 41, 845-850.

30. Hodge AM, Simpson JA, Gibson RA, et al. (2007) Plasma phospholipid fatty acid composition as a biomarker of habitual dietary fat intake in an ethnically diverse cohort. Nutr Metab Cardiovasc Dis 17, 415-426.

31. Andersen LF, Solvoll K, Johansson LRK, et al. (1999) Evaluation of a food frequency questionnaire with weighed records, fatty acids, and alpha-tocopherol in adipose tissue and serum. Am J Epidemiol 150, 75-87.

32. Sanders TA (2000) Polyunsaturated fatty acids in the food chain in Europe. Am J Clin Nutr 71, 176S-178S.

33. Arab L (2003) Biomarkers of fat and fatty acid intake. J Nutr 133, 925S-932S.

34. Kobayashi M, Sasaki S, Kawabata T, et al. (2001) Single measurement of serum phospholipid fatty acid as a biomarker of specific fatty acid intake in middle-aged Japanese men. Eur J Clin Nutr 55, 643-650.

35. Hunter DJ, Rimm EB, Sacks FM, et al. (1992) Comparison of measures of fatty acid intake by subcutaneous fat aspirate, food frequency questionnaire, and diet records in a free-living population of US men. Am J Epidemiol 135, 418-427.

36. Tjonneland A, Overvad K, Thorling E, et al. (1993) Adipose tissue fatty acids as biomarkers of dietary exposure in Danish men and women. Am J Clin Nutr 57, 629-633.

37. Connor SL, Zhu N, Anderson GJ, et al. (2000) Cheek cell phospholipids in human infants: a marker of docosahexaenoic and arachidonic acids in the diet, plasma, and red blood cells. Am J Clin Nutr 71, 21-27.

38. Zhang B, Wang P, Chen C-G, et al. (2010) Validation of an FFQ to estimate the intake of fatty acids using erythrocyte membrane fatty acids and multiple $3 \mathrm{~d}$ dietary records. Public Health Nutr 13, 1546-1552.

39. Swierk M, Williams PG, Wilcox J, et al. (2011) Validation of an Australian electronic food frequency questionnaire to measure polyunsaturated fatty acid intake. Nutrition $\mathbf{2 7}$, 641-646.

40. Shahbakhti H, Watson REB, Azurdia RM, et al. (2004) Influence of eicosapentaenoic acid, an omega-3 fatty acid, on ultraviolet-B generation of prostaglandin-E2 and proinflammatory cytokines interleukin- $1 \beta$, tumor necrosis factor- $\alpha$, 
interleukin-6 and interleukin-8 in human skin in vivo. Photochem Photobiol 80, 231-235.

41. Rhodes LE, Belgi G, Parslew R, et al. (2001) UltravioletB-induced erythema is mediated by nitric oxide and prostaglandin E2 in combination. J Invest Dermatol 117, 880-885.

42. van der Pols JC, Xu C, Boyle GM, et al. (2011) Serum omega3 and omega- 6 fatty acids and cutaneous p53 expression in an Australian population. Cancer Epidemiol Biomarkers Prev 20, 530-536.

43. Garbe C \& Leiter U (2009) Melanoma epidemiology and trends. Clin Dermatol 27, 3-9.

44. Kristal AR, Peters U \& Potter JD (2005) Is it time to abandon the food frequency questionnaire? Cancer Epidemiol Biomarkers Prev 14, 2826-2828. 